\title{
STAR FORMATION: CAN THERE BE A BREAK IN THE IMF NEAR 0.1M $\odot$ ?
}

\author{
TAKENORI NAKANO \\ Nobeyama Radio Observatory, National Astronomical Observatory \\ Nobeyama, Minamimaki, Minamisaku, Nagano 384-13, Japan
}

\section{Introduction}

The initial mass function of stars (IMF) at small masses depends on several factors. First, it depends on the mass function of cloud cores in which stars form. Second, there must be a lower limit to the core mass for contraction; very small mass cores may not contract even if they exist. This must affect greatly the IMF near its lower end. Third, not all core matter may become stars; we must determine the stellar mass $M_{\star}$, or the star formation efficiency $M_{\star} / M_{\mathrm{cc}}$, as a function of the mass of the cloud core, $M_{\mathrm{cc}}$. In this paper we discuss the second and third points.

\section{Critical Core Mass for Contraction}

There is a widely accepted assumption that low-mass stars form in magnetically subcritical cloud cores and high-mass stars form in magnetically supercritical ones (e.g., review by Shu, Adams, \& Lizano 1987); the magnetic force in magnetically supercritical cores is not strong enough to support the cores against the self-gravity, and subcritical cores can be in magnetohydrostatic equilibrium only when their expansion by the magnetic force (diluted by the gravity) is controlled by the external magnetic field and/or external pressure. However, Nakano (1998) showed that cloud cores, which are generally observed as portions of a molecular cloud having higher column density than the surrounding, are magnetically supercritical.

For a magnetically supercritical cloud core there is a critical value $P_{\text {cr }}$ for the surface pressure $P_{\mathrm{s}}$, above which no magnetohydrostatic equilibria exist and the core collapses, given by (Nakano 1998)

$$
\frac{P_{\mathrm{cr}}}{k} \approx 7.1 \times 10^{4} \frac{1}{a_{\mathrm{eff}}^{4}}\left(\frac{M_{\mathrm{cc}}}{1 M_{\odot}}\right)^{-2}\left(\frac{C_{\mathrm{eff}}}{0.19 \mathrm{~km} \mathrm{~s}^{-1}}\right)^{8} \mathrm{~cm}^{-3} \mathrm{~K}
$$

where $k$ is the Boltzmann constant, $C_{\text {eff }}$ is the effective sound velocity in the core including the effect of turbulence, and $a_{\text {eff }} \approx 1-\left(\Phi / \Phi_{\mathrm{cr}}\right)^{2}$ is the effective coefficient for the gravity diluted by the magnetic force; $\Phi$ and $\Phi_{\mathrm{cr}} \approx 2 \pi G^{1 / 2} M_{\mathrm{cc}}$ are the magnetic flux and the critical magnetic flux, respectively, of the core, $G$ is the gravitational constant, and $\Phi<\Phi_{\text {cr }}$ for a magnetically supercritical core.

Because the surface pressure $P_{\mathrm{s}}$ on the cores is nearly equal to the mean pressure in the cloud in which the cores are embedded unless they are located close to the cloud surface, we have

$$
\frac{P_{\mathrm{s}}}{k} \approx \frac{G \Sigma^{2}}{k} \approx 8.0 \times 10^{4}\left(\frac{A_{V}}{3 \mathrm{mag}}\right)^{2} \mathrm{~cm}^{-3} \mathrm{~K}
$$

where $\Sigma$ and $A_{V}$ are the column density and the extinction at visible, respectively, of the cloud.

Although $C_{\text {eff }}$ can hardly be less than $0.19 \mathrm{~km} \mathrm{~s}^{-1}$ corresponding to a temperature of $10 \mathrm{~K}$ even without turbulence, cloud cores of $M_{\mathrm{cc}} \gtrsim 0.3 M_{\odot}$ can contract if they are embedded in ordinary molecular clouds with $A_{V} \sim 10 \mathrm{mag}$ as seen by comparing equations (1) and (2). 


\section{Star Formation Efficiency}

Dynamical contraction of a cloud core is highly nonhomologous. A small protostellar core in quasiequilibrium forms first, and this core grows gradually by inflow of matter from the outer part of the cloud core. The mass of a forming star is fixed when the mass inflow is stopped.

As a mechanism of stopping accretion onto protostars many people considered the reversion of inflows by stellar winds (e.g., review by Shu et al. 1987). However, only a part of the inflow is reversed as seen from the shapes of bipolar outflows.

Nakano, Hasegawa, \& Norman (1995) proposed a different mechanism that determines the mass of the star. When a significant fraction of the core matter is blown off by the mass outflows from the forming star, the remaining matter is no longer gravitationally bound unless the initial core mass is much larger than the critical mass for contraction, and disperses. In this way the forming star stops growing. With some simple modifications to the theory of Nakano et al. (1995) the star formation efficiency is given by

$$
\begin{gathered}
\frac{M_{\star}}{M_{\mathrm{cc}}} \approx 0.043\left(\frac{\dot{M}_{\mathrm{w}} / \dot{M}_{\mathrm{I}}}{0.1} \frac{V_{\mathrm{w}}}{200 \mathrm{~km} \mathrm{~s}^{-1}}\right)^{-1 / 2}\left(\frac{\Omega_{\mathrm{w}}}{4 \pi}\right)^{1 / 2}\left(\frac{1-\dot{M}_{\mathrm{w}} / \dot{M}_{\mathrm{I}}}{0.9}\right) \\
\times\left(\frac{M_{\mathrm{cc}}}{100 M_{\odot}}\right)^{1 / 6}\left(\frac{n_{\mathrm{cc}}}{10^{4} \mathrm{~cm}^{-3}}\right)^{1 / 12}+\frac{M_{0}}{M_{\mathrm{cc}}},
\end{gathered}
$$

where $V_{\mathrm{w}}$ is the velocity of the wind near its base, $\dot{M}_{\mathrm{I}}$ is the mass inflow rate to the star, $\dot{M}_{\mathrm{w}}$ is the mass loss rate by the wind, $\Omega_{\mathrm{w}}$ is the solid angle of the outflows seen from the star, $n_{\mathrm{cc}}$ is the mean density of the cloud core, and $M_{0}$ is the mass of the star when it begins to drive the wind. For $M_{\star} \gg M_{0}$ the star formation efficiency is rather insensitive to $M_{\mathrm{cc}}$ and $n_{\mathrm{cc}}$ and is $10 \%$ on the order of magnitude.

\section{Onset of Mass Outflows}

Winds from protostars are essential in determining the stellar mass except for very high mass stars where expansion of HII regions may be important (Nakano et al. 1995). At what mass do protostars begin to drive winds? Although the mechanism of driving bipolar outflows from young stellar objects is not well known, we have an example of the outflows from a seemingly very low mass protostar. André, Ward-Thompson, \& Barsony (1993) estimated the mass of the protostar driving the bipolar flows in VLA 1623 as less than $0.4 M_{\odot}$. They also argued a possibility that the mass is less than $0.03 M_{\odot}$. Because brown dwarfs have effective temperature as high as $3000 \mathrm{~K}(e . g$., Hayashi \& Nakano 1963; D'Antona \& Mazzitelli 1985, 1994), which is nearly equal to that of T Tauri stars, the ionization fraction in the atmosphere is not much different from that of $T$ Tauri stars. In addition mass accretion is going on at the surface. Therefore, we can expect magnetic activities and mass outflows from mass-accreting stars with mass much smaller than $0.1 M_{\odot}$, i.e., $M_{0}$ in equation (3) may be much smaller than $0.1 M_{\odot}$.

\section{Summary}

We have found that cloud cores of mass as low as $0.3 M_{\odot}$ can contract. We can expect mass outflows even from protostars of $M_{\star} \ll 0.1 M_{\odot}$. The star formation efficiency is of the order of $10 \%$ at $M_{\star} \gg M_{0}$. Therefore, we can expect formation of stars whose mass is significantly smaller than $0.1 M_{\odot}$. Thus, from the theory of star formation we have found no clear evidences for the existence of a break in the IMF at $M_{\star} \approx 0.1 M_{\odot}$ unless there is a break in the mass function of cloud cores at $M_{\mathrm{CC}} \sim 1 M_{\odot}$.

\section{References}

André, P., Ward-Thompson, D., Barsony, M. 1993, ApJ, 406, 122.

D'Antona, F., Mazzitelli, I. 1985, ApJ, 296, 502.

D'Antona, F., Mazzitelli, I. 1994, ApJS, 90, 467.

Hayashi, C., Nakano, T. 1963, Prog. Theor. Phys., 30, 460.

Nakano, T. 1998, ApJ, in press.

Nakano, T., Hasegawa, T., Norman, C. 1995, ApJ, 450, 183.

Shu, F.H., Adams, F.C., Lizano, S. 1987, ARAA, 25, 23. 